\title{
Genetic Variability and Marker Trait Association analysis of Various Phenological and Yield Related Traits for Heat Tolerance in Chickpea (Cicer arietinum L.)
}

\author{
Uday Chand Jha ${ }^{1,2^{*}}$, Paresh Chandra Kole ${ }^{2}$, Narendra Pratap Singh ${ }^{1}$ \\ ${ }^{1}$ Indian Institute of Pulses Research (IIPR), Kanpur, Uttar Pradesh (208 024), India \\ ${ }^{2}$ Dept. of Genetics \& Plant Breeding and Crop Physiology, Institute of Agriculture, Visva-Bharati, Sriniketan, Bolpur, West \\ Bengal (731 236), India
}

\section{Corresponding Author}

Uday Chand Jha

e-mail: uday_gene@yahoo.co.in

\section{Article History}

Article ID: AR1872

Received in $21^{\text {st }}$ April, 2018

Received in revised form 19 ${ }^{\text {th }}$ May, 2018

Accepted in final form $4^{\text {th }}$ June, 2018

\begin{abstract}
Increasing incidence of heat stress (HS) is receiving serious attention as it causes significant yield reduction in various crops including chickpea worldwide. Here, we investigated the existing genetic variability for various yield related crucial traits for developing heat tolerant genotype under field condition in a panel of seventy eight chickpea genotypes under normal and HS condition via conducting augmented design analysis. Analysis of variance (ANOVA) exhibited significance difference among the checks for first flowering (FF), days to $50 \%$ flowering (50F), days to pod initiation (DPI), days to maturity (MAT), plant height (PH), empty pod (EP), yield plant ${ }^{-1}$ (YPP), biological yield (BioY), harvest index (HI\%), and 100 seed weight (100 SW) under normal condition. While, under HS condition significance difference among the checks for the following traits FF, 50F, PH, EP, YPP and 100SW were recorded. Additionally, to seek marker trait association (MTA), we examined MTAs for the given phenological and yield related traits under both normal and late sown condition via employing 81 simple sequence repeat (SSR) markers in the given set of genotypes. A total of 37 significant MTAs (under normal condition) and 38 significant MTAs (under HS condition) were obtained for various phenological and yield related traits. Additionally, eighteen MTAs for heat tolerance index (HTI), stress susceptibility index (SSI), yield index (YI), mean productivity (MP) and geometric mean productivity (GMP) were recorded.
\end{abstract}

Keywords: Genetic variability, heat stress, MTA, SSR

\section{Introduction}

Chickpea remains as an important cool season global grain legume crop, offering plant based dietary protein and essential micro nutrients to human population across the globe (Graham and Vance, 2003). Chickpea stands as the second most important global grain legume next to common bean (FAO, 2014), contributing $14.2 \mathrm{mt}$ to the global food basket from $14.8 \mathrm{mha}$ area across the globe with an average productivity of $0.96 \mathrm{tha}^{-1}$ (FAO, 2014). However, chickpea yield is seriously challenged by various biotic and abiotic stresses impeding to attain its potential yield (Jha et al., 2014a). In parallel, given the current deleterious effects of global climate change, increasing event of heat stress (HS) is appearing as one of the important abiotic stresses, causing detrimental effects on various crops including cool season grain legumes (Jha et al., 2014a, 2014b ; Jha et al., 2017). Significant phenological changes and detrimental effects on pre- and post reproductive processes leading to reduction in yield have been recorded in chickpea under terminal HS (Devasirvatham et al., 2013; Jha et al., 2015; Krishnamurthy et al., 2011).
Given the increase in $1{ }^{\circ} \mathrm{C}$ "seasonal temperature" in North India during chickpea growing season may lead to reduction of $53 \mathrm{~kg} \mathrm{ha}^{-1}$ yield in chickpea (Kalra et al., 2008). Therefore, breeding for heat tolerance in chickpea is urgently needed to sustain chickpea yield under the increasing incidences of HS. In the context, conventional breeding driven efforts have enabled in identification of ICC 92944, ICC 1205, ICC 4958 chickpea genotypes as source of HS tolerance under field condition (Devasirvatham et al., 2013; Krishnamurthy et al., 2011). However, progress in development in designing HS tolerant chickpea remains slow. In parallel surge of various advanced molecular markers have offered great opportunity to the breeder community to exploit them in marker assisted breeding scheme for improving various complex traits including HS in chickpea (Bajaj et al., 2015a; Thudi et al., 2014; Varshney et al., 2014; Kale et al., 2015; Jha et al., 2018). Thus, role of (MTA) analysis an approach of marker assisted molecular breeding could be of great importance for identifying genomic regions conferring HS tolerance thereby, accelerating the HS tolerance breeding in chickpea. Here, we investigated the existing genetic variability for phenological and yield related 
traits in a panel of seventy eight chickpea genotypes (including historically high yielding released varieties in India, improved breeding lines and accessions) under normal and late sown condition. Additionally, we examined the MTAs for various yield related traits under both normal and late sown condition via employing 81 simple sequence repeat (SSR) markers in the 71 genotypes for facilitating marker assisted breeding for HS tolerance in chickpea.

\section{Materials and Methods}

\subsection{Experimental material}

The experimental material constituted 78 chickpea genotypes containing historically released varieties cultivated across the India, accessions from ICRISAT, Patancheru, improved breeding lines of Indian Institute of Pulses Research (IIPR), Kanpur and JNKVV Jabalpur including three heat tolerant checks (ICC 1205, ICC 4958 and ICC 92944) (Devasirvatham et al., 2012; Devasirvatham et al., 2013). The crop was grown in the second week of November 2105 (normal sown) and the late sown crop was grown in second week of January 2016 (HS) at the main farm of Indian Institute of Pulses Research (IIPR), Kanpur. The average weekly temperature recorded during the crop growth period from $2^{\text {nd }}$ week of November 2015 to April 2016 is given in (Figure 1). Each genotype was sown in two rows having $4 \times 0.3 \mathrm{~m}^{2}$ plot size. All the 75 genotypes were planted in augmented design having 5 blocks with the above given three checks replicated in each blocks. Randomly five plants of each genotype were selected. Average data of five plants for each genotype was recorded for first flowering (FF), $50 \%$ flowering (50F), days to pod initiation (DPI), days to pod filling (DPF), plant height (PH), days to maturity (MAT), primary

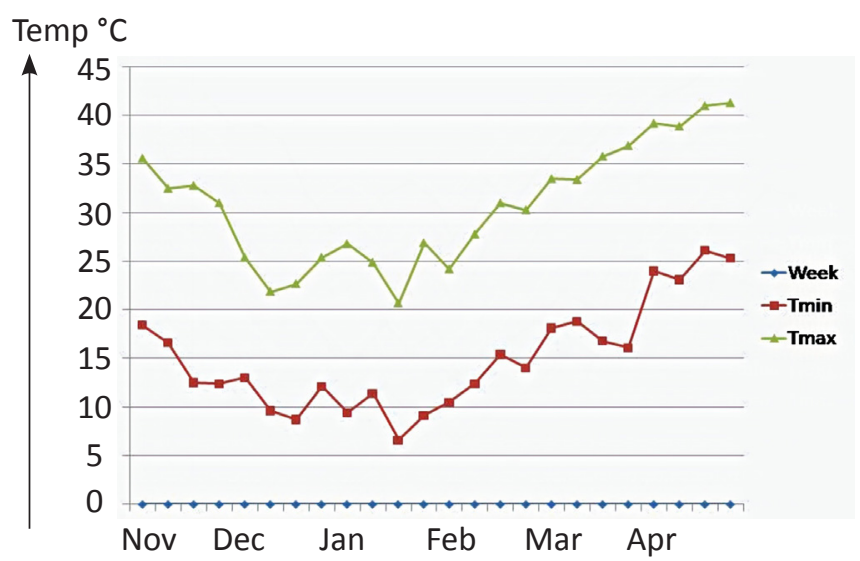

Figure 1: Mean weekly minimum and maximum day temperature recorded during the crop season in 2015-2016

branches (PB), biological yield (Bio Y), number of pods plant ${ }^{-1}$ (NOPS), empty pods plant ${ }^{-1}(E P)$, yield plant ${ }^{-1}$ (YPP), harvest index \% (HI\%), 100 seed weight (100 seed Wt) and Plot Yield (PY) various traits of breeding interest under both conditions.

\subsection{Statistical analysis}

ANOVA for the given data in augmented design (Federer, 1956) was analyzed by using R software. Additionally, to seek
MTAs with the heat tolerance indices, we also estimated yield stability index $\mathrm{HTI}, \mathrm{YI}, \mathrm{SSI}, \mathrm{MP}$ and GMP five important heat tolerance indices. The indices were calculated as per the suggested formulae below.

SSI=(1-(Ysi/Ypi))/SI (Fischer and Maurer, 1978)

$\mathrm{HTI}=(\mathrm{Ys} \times \mathrm{Yp}) / \mathrm{Yp}^{2}$ (Fernandez, 1992)

Yield index (YI)=Ys/Yp (Bouslama and Schapaugh, 1984)

Mean productivity (MP) $=(Y p i+Y s i) / 2$ Hossain et al. (1990)

Geometric mean productivity $(\mathrm{GMP})=\vee$ Ypi $\times$ Ysi $($ Ramirez and Kelly, 1998)

Ysi and Ypi are the mean grain yield of individual genotype in HS and non HS conditions; whereas, Ys denote the mean yield of genotype under HS and $\mathrm{Yp}$ the mean yield of genotype under normal condition.

\subsection{DNA extraction and SSR analysis}

As per the CTAB method suggested by Doyel and Doyle (1987) genomic DNA was extracted from 71 chickpea genotypes. Given the screening of 120 SSR markers in the given set of genotypes, a total of 81SSRs yielded polymorphic fragments. The SSR markers used here are reported previously by different research groups Winter et al. (1999, 2000); Sethy et al. (2003); Sethy et al. (2006); Gaur et al. (2011); Choudhary et al. (2009); Choudhary et al. (2012) existing across the all eight linkage groups in chickpea.

\subsection{PCR analysis}

The PCR assay was carried out in a $10 \mu$ reaction mixture containing $5.9 \mu \mathrm{l}$ of sterilized distilled water, $1.00 \mu \mathrm{l}$ template DNA ( $25 \mathrm{ng}$ ), $0.5 \mu \mathrm{l}$ of forward and $0.5 \mu \mathrm{l}$ of reverse primer ( 5 $\mu \mathrm{M}), 1.00 \mu \mathrm{l}$ 10×PCR buffer (10 mMTris-Hcl, $50 \mathrm{mMKcl}, \mathrm{pH} 8.3$ ), $1.00 \mu \mathrm{l}$ dNTP mix (0.2 mM each of dATP, dGTP, dCTP and dTTP)

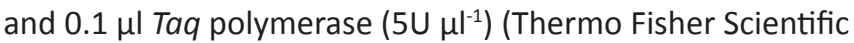
Mumbai, India, Pvt. Ltd.) by usingG-40402 thermo cycler (G-STORM, Somerset, UK). A touch down PCR profile was used for amplifications with initial denaturation at $94{ }^{\circ} \mathrm{C}$ for $5 \mathrm{~min}$ followed by 10 cycles of touch down $61-51^{\circ} \mathrm{C}, 30 \mathrm{~s}$ at $94^{\circ} \mathrm{C}$, annealing for $30 \mathrm{~s}$ at $61^{\circ} \mathrm{C}$ (the annealing temperature for each cycle being reduced by $1^{\circ} \mathrm{C}$ per cycle) and extension for $30 \mathrm{~s}$ at $72{ }^{\circ} \mathrm{C}$. This was accompanied by 40 cycle of denaturation at $94{ }^{\circ} \mathrm{C}$ for $30 \mathrm{~s}$, annealing at $51{ }^{\circ} \mathrm{C}$ for $30 \mathrm{~s}$, elongation at 72 ${ }^{\circ} \mathrm{C}$ for $45 \mathrm{~s}$, and $10 \mathrm{~min}$ of final extension at $72^{\circ} \mathrm{C}$. Amplified fragments were resolved in $3 \%$ agarose gel using $0.5 \times \mathrm{TBE}$ running buffer and images were analyzed with Quantity one software (Bio-Rad, CA 94547, USA).

\subsection{Marker-trait association analysis}

The phenotypic data on fourteen traits and the genotypic data were analyzed to examine significant MTAs. Here we employed mixed linear model (MLM) based on Q+K matrix. We used TASSEL v. 3.0 (Bradbury et al., 2007; Zhang et al., 2010) to detect MTAs, and $p=0.05$ and $p=0.01$ were considered as a significance threshold. 


\section{Results and Discussion}

\subsection{Genetic variability}

General statistics for various traits recorded under both normal and late sown trials are given in (Table 1). Mean square for analysis of variance (ANOVA) suggested significant difference among all the checks for most of the traits except (PB, DPF, BioY plant ${ }^{-1}$ and $\mathrm{HI} \%$ ) under normal sown condition (see Table 2). While, under HS condition significant difference among the checks for the following traits FF, 50F, PH, EP, BioY/P,YPP,

\begin{tabular}{lccccccccccccccc}
\hline \multicolumn{10}{l}{ Table 1: Genetic variability studied for various traits in the genotypes } \\
\hline Traits & $\begin{array}{c}\text { FF } \\
\text { (days) }\end{array}$ & $\begin{array}{c}\text { 50F } \\
\text { (days) }\end{array}$ & $\begin{array}{c}\text { DPI } \\
\text { (days) }\end{array}$ & $\begin{array}{c}\text { DPF } \\
\text { (days) }\end{array}$ & $\begin{array}{c}\text { PH } \\
\text { (cm) }\end{array}$ & $\begin{array}{c}\text { MAT } \\
\text { (days) }\end{array}$ & $\begin{array}{c}\text { PB } \\
\text { (dio Y } \\
\text { (g) }\end{array}$ & NOPS & EP & $\begin{array}{c}\text { YPP } \\
\text { (g) }\end{array}$ & HI\% & $\begin{array}{c}100 \text { S } \\
\text { W (g) }\end{array}$ & PY (g) \\
\hline Min & 37 & 43 & 52 & 62 & 38.6 & 98 & 4 & 8.9 & 31 & 2 & 6.1 & 11 & 7.1 & 75 \\
Max & 82 & 86 & 92 & 115 & 64.3 & 143 & 6 & 49.7 & 139 & 8 & 18.6 & 78.24 & 35.9 & 390 \\
Mean & 62.00 & 70.00 & 76.00 & 88.00 & 49.42 & 130.00 & 5.00 & 22.15 & 61.00 & 5.00 & 13.23 & 43.80 & 18.20 & 277.78 \\
SE & 2.17 & 2.48 & 3.04 & 4.80 & 3.00 & 1.96 & 0.73 & 0.71 & 9.48 & 0.48 & 0.66 & 3.15 & 1.08 & 9.370 \\
CV \% & 3.48 & 3.52 & 4.01 & 5.50 & 6.07 & 1.50 & 14.80 & 3.21 & 15.40 & 8.80 & 5.05 & 7.21 & 5.94 & 3.37 \\
Late sown trial in 2016 & & & & & & & & & & & & \\
\hline Min & 29 & 37 & 40 & 56 & 21.3 & 81 & 3 & 5.8 & 11 & 3 & 3.5 & 28.1 & 7.3 & 42 \\
Max & 59 & 68 & 78 & 95 & 39.3 & 112 & 6 & 13.5 & 39 & 15 & 6.2 & 78 & 24.8 & 234 \\
Mean & 48.00 & 55.00 & 63.00 & 76.00 & 30.10 & 98.00 & 4.00 & 9.93 & 22.00 & 7.00 & 4.60 & 49.00 & 14.50 & 141.00 \\
SE & 2.42 & 3.80 & 6.49 & 5.05 & 0.97 & 2.30 & 0.74 & 0.48 & 3.01 & 0.77 & 0.20 & 6.13 & 0.80 & 3.700 \\
CV \% & 5.00 & 6.87 & 10.38 & 6.68 & 3.25 & 2.48 & 17.65 & 4.91 & 13.64 & 10.37 & 4.41 & 12.53 & 5.54 & 2.67 \\
\hline
\end{tabular}

Table 2: Analysis of variance for normal sown trial (2015-16)

\begin{tabular}{lllllllllllll}
\hline \multicolumn{10}{c}{ Mean squares } \\
\hline Source & df & FF & $50 F$ & DPI & DPF & PH & DM & PB & Bio Yplant & NOPS & EP & YPP \\
\hline Blocks & 4 & 1.23 & 3.23 & 9.56 & 10.06 & 13.23 & 8.266667 & 0.23 & 13.83 & 75.76 & 0.43 & 0.07 \\
Trt & 77 & $88.34^{* *}$ & $84.47^{* *}$ & $61.13^{* *}$ & $75.95^{*}$ & $31.86^{*}$ & $55.11^{* *}$ & 0.43 & $36.2^{*}$ & $327.46^{*}$ & $1.43^{* *}$ & $9.07^{* *}$ \\
Tests & 74 & $72.13^{* *}$ & $73.91^{* *}$ & $58.76^{* *}$ & $76.37^{*}$ & $31.18^{*}$ & $50.974^{* *}$ & 0.37 & 18.49 & $334.34^{*}$ & $1.38^{* *}$ & $5.14^{* *}$ \\
checks & 2 & $154.4^{* *}$ & $133.26^{* *}$ & $122.6^{* *}$ & 91.46 & $63.72^{*}$ & $231.2^{* *}$ & 1.86 & 12.74 & 212.46 & $1.06^{* *}$ & $4.27^{* *}$ \\
Test vs check & 1 & $1155.2^{* *}$ & $768.32^{* *}$ & $113.5^{* *}$ & 13.86 & 18.52 & $9.38^{* *}$ & 2 & $1393.56^{* *}$ & 48.02 & $6.48^{* *}$ & $309.5^{* *}$ \\
Error & 8 & 4.73 & 49.46 & 9.26 & 23.46 & 9.02 & 3.86 & 0.53 & 9.3 & 89.96 & 0.233 & 0.44 \\
\hline
\end{tabular}

Table 2: Continue...

\begin{tabular}{lccc}
\hline \multicolumn{4}{c}{ Mean squares } \\
\hline Source & HI \% & $100 \mathrm{~S} \mathrm{~W}$ & PY \\
\hline Blocks & 30.08 & 1.27 & 60.11 \\
Trt & 19.96 & $36.89^{* *}$ & $5113.18^{* *}$ \\
Tests & 19.56 & $29.19^{* *}$ & $4323.14^{* *}$ \\
checks & 12.68 & $253.21^{* *}$ & $11557.59^{* *}$ \\
Test vs check & 64.02 & $173.47^{* *}$ & $50687.15^{* *}$ \\
Error & 13.07 & 1.17 & 87.9 \\
\hline
\end{tabular}

100SW and PY were recorded (Table 3). In this connection Jha et al. (2015), Jha and Shil (2015), and Krishnamurthy et al. (2011) recorded significant genetic variability for different phenological and yield related traits under HS in chickpea. Thus, genotype having high BioY, $\mathrm{HI}$ and 100SW under HS could be potentially incorporated into chickpea breeding programme for transferring these traits to the HS tolerant yet low 100 SW genotypes for sustaining yield under HS. Moreover, the tested genotypes could be introduced as parent in crossing programme for developing HS tolerance in chickpea.

\subsection{MTA analysis}

MTA study is gaining enormous attention from marker assisted breeding point of view in various crop plants including chickpea (Bajaj et al., 2015a; Thudi et al., 2014). Notable instances of MTA study for investigating genomic regions related to drought stress tolerance traits have been recorded (Jamalabadi et al., 2013; Kale et al., 2015; Thudi et al., 2014; Varshney et al., 2014). However, MTA for HS tolerance in chickpea is limitedly exploited (Thudi et al., 2014; Jha et al., 2018).

In the current study a total of 37 significant MTAs under 
Jha et al., 2018

\begin{tabular}{|c|c|c|c|c|c|c|c|c|c|c|c|c|}
\hline \multicolumn{13}{|c|}{ Mean squares } \\
\hline Source & df & $\mathrm{FF}$ & $50 \mathrm{~F}$ & DPI & DPF & $\mathrm{PH}$ & $\mathrm{DM}$ & PB & $\begin{array}{l}\text { Bio Y } \\
\text { plant }\end{array}$ & NOPS & EP & YPP \\
\hline Blocks & 4 & 2.06 & 3.9 & 17.93 & 10.73 & 1.05 & 4.4 & 0.01 & 0.09 & 1.06 & 0.5 & 0.027 \\
\hline Trt & 77 & $52.94^{* *}$ & $51.39^{*}$ & 50.53 & 38.32 & $13.69^{* *}$ & $34.27^{* *}$ & 0.49 & $2.34^{*}$ & $63^{* *}$ & $5.89^{* *}$ & $0.42^{* *}$ \\
\hline Tests & 74 & $36.92^{* *}$ & 42.25 & 49.89 & 38.26 & $7.5^{* *}$ & $30.19^{* *}$ & 0.44 & $1.67^{*}$ & $30.69^{*}$ & $5.59^{* *}$ & $0.39^{* *}$ \\
\hline checks & 2 & $375.2^{* *}$ & $261.66^{* *}$ & 63.26 & $52.86 \quad 5$ & $53.94^{* *}$ & 16.46 & 0.46 & $1.41^{\circ}$ & 36.86 & $8.26^{* *}$ & $1.26^{* *}$ \\
\hline Test vs check & 1 & $593.97^{* *}$ & $307.52^{* *}$ & 72.8 & $14.22 \quad 39$ & $391.25^{* *}$ & $371.73^{* *}$ & 3.92 & 53.32 & $2506.32^{* *}$ & $2^{* *} 23.12^{* *}$ & $0.72^{* *}$ \\
\hline Error & 8 & 5.86 & 14.5 & 337.46 & 204.26 & 7.68 & 42.4 & 0.55 & 0.23 & \multicolumn{2}{|c|}{9.11} & 0.04 \\
\hline \multicolumn{6}{|c|}{ Table 3: Continue... } & \multicolumn{7}{|c|}{ Table 4: MTA analysis for trial 2015-16 (Normal sown) } \\
\hline \multicolumn{6}{|c|}{ Mean squares } & \multirow{2}{*}{$\begin{array}{l}\text { Trait } \\
\text { FF }\end{array}$} & \multicolumn{3}{|c|}{ Marker name } & LG & $P$ value & \multirow{2}{*}{$\begin{array}{c}\text { PV\% } \\
19\end{array}$} \\
\hline Source & & HI \% & $100 s$ & & PY & & CESS & R 159 & & LG 3 & $0.00007^{* *}$ & \\
\hline $\begin{array}{l}\text { BlOCKS } \\
\text { Trt }\end{array}$ & & 1.51 & 1.0 & & 13.2 & $\mathrm{FF}$ & CESS & R 43 & & LG 4 & $0.03814^{*}$ & 6.3 \\
\hline Tests & & $\begin{array}{l}20.45 \\
16.92^{* *}\end{array}$ & 12. & & $\begin{array}{l}1261.02 \\
1178.11^{* *}\end{array}$ & $\mathrm{FF}$ & $\mathrm{NCPG}$ & R 41 & & LG 7 & $0.04066^{*}$ & 6.4 \\
\hline checks & & $95.81^{* *}$ & 49.1 & & $308.46^{* *}$ & $50 \mathrm{~F}$ & CESSI & R 159 & & LG 3 & $0.00005^{* *}$ & 19.7 \\
\hline Test vs check & & $131.19^{* *}$ & 239. & & $9301.57^{* *}$ & $50 \mathrm{~F}$ & CakTp & OSSRO3 & 637 & LG 4 & $0.0443^{*}$ & 17.3 \\
\hline Error & & 37.69 & 0.6 & & 14.21 & $50 \mathrm{~F}$ & CESS & R 43 & & LG 4 & $0.04981^{*}$ & 5.6 \\
\hline${ }^{*} p=0.05$ and & $\beta$ & & & & & $50 \mathrm{~F}$ & CESS! & R 45 & & LG 4 & $0.03709^{*}$ & 6.5 \\
\hline & & & & & & DPF & CESSI & R 159 & & LG 3 & $0.01706^{*}$ & 8 \\
\hline $\begin{array}{l}\text { normal conditi } \\
\text { HS condition ( }\end{array}$ & on & ee Table 4) & various & rec & $\begin{array}{l}\text { As under } \\
\text { aits have }\end{array}$ & DPI & CESS & R 159 & & LG 3 & $0.00935^{* *}$ & 10.4 \\
\hline been recorde & d. Co & ncurrently & , eighte & MTAs & for various & MAT & CESS & R 45 & & LG 4 & $0.00658^{* *}$ & 11.4 \\
\hline HS related inc & ices & have beer & noted (s & ee Tabl & le 6). MTAs & MAT & TR7 & & & LG7 & $0.02^{*}$ & 11 \\
\hline distribution an & d q & antile-qua & ntile $(Q-Q$ & ) plots & are depicted & PB & NCPE & R231 & & LG3 & $0.039^{*}$ & 15.4 \\
\hline $\begin{array}{l}\text { in Figure } 2 \text { (fo } \\
\text { in Figure } 4 \text { (fo }\end{array}$ & $\begin{array}{l}\text { norr } \\
\text { r hea }\end{array}$ & $\begin{array}{l}\text { mal sown), } \\
\text { t toleranc }\end{array}$ & $\begin{array}{l}\text { in Figure } \\
\text { e indices) }\end{array}$ & $\begin{array}{l}3 \text { (for H } \\
\text { conside }\end{array}$ & $\begin{array}{l}\text { IS sown) and } \\
\text { ering MLM }\end{array}$ & PB & GA10 & & & LG5 & $0.02339^{*}$ & 7.6 \\
\hline model. The ma & kers & witnessed & significant & associa & tion with the & PB & NCPG & R12 & & LG6 & $0.03608^{*}$ & 7.2 \\
\hline given traits by & devia & ting from $n$ & ull expect & ation de & picted in QQ & BioY & GA9 & & & LG5 & $0.03394^{*}$ & 18.4 \\
\hline $\begin{array}{l}\text { plots. Phenol } \\
\text { selection of } \mathrm{HS}\end{array}$ & gical & traits rem & lain crucia & I for ev & $\begin{array}{l}\text { aluation and } \\
\text { ing chickpea }\end{array}$ & BioY & NGPC & SR225 & & LG6 & $0.01952^{*}$ & 11.9 \\
\hline (Devasirvatha & $\mathrm{m}$ et & al., 2013 & ). Taking & note of & f this, three & BioY & NCPE & R56 & & LG1 & $0.046^{*}$ & 8 \\
\hline significant MT & Is on & LG3, LG4 & and on LG & 7 for $F F$ & traits under & NOPS & STMS & & & LG6 & $0.03768^{*}$ & 12.7 \\
\hline normal condit & on at & nd one sigr & nificant $M$ & TA on $L C$ & G6 under HS & NOPS & NCPE & R200 & & LG2 & $0.04^{*}$ & 16 \\
\hline for FF trait was & reco & ded on LG & 3 and LG4 & ender dr & rought stress & EP & GA6 & & & LG6 & $0.04081^{*}$ & 23.1 \\
\hline (Rehman et al., & 201 & ). In this co & ntext Jam & alabadi & et al. (2013) & EP & NCPG & R2O2 & & LG2 & $0.04^{*}$ & 17.1 \\
\hline also reported & one c & losely linke & d marker & with FF & on LG3. For & EP & $\mathrm{H} 2 \mathrm{BO}$ & & & LG4 & $0.045^{*}$ & 4 \\
\hline $\begin{array}{l}50 \mathrm{~F} \text { trait, a tot } \\
\text { explaining up } \mathrm{t}\end{array}$ & $\begin{array}{l}\text { al of } \\
\text { o } 19 \text {. }\end{array}$ & $\begin{array}{l}\text { four signifi } \\
7 \text { PV\%, whi }\end{array}$ & $\begin{array}{l}\text { cant MTAs } \\
\text { le, two sig }\end{array}$ & $\begin{array}{l}\text { on LG4 } \\
\text { ificant }\end{array}$ & $\begin{array}{l}4 \text { and on LG3 } \\
\text { MTAs for the }\end{array}$ & YPP & H1BO & & & LG5 & $0.04538^{*}$ & 5 \\
\hline same trait on & G6 & vas record & ed under I & ate sow & n condition. & YPP & NCPC & R234 & & LG 6 & $0.03318^{*}$ & 18.5 \\
\hline Considering $\mathrm{D}$ & I anc & DPF traits & , significan & t MTA n & was noted on & YPP & $\mathrm{H} 5 \mathrm{AO}$ & & & LG 7 & $0.0383^{*}$ & 15.4 \\
\hline $\begin{array}{l}\text { LG3, whereas t } \\
\text { MTAs (for DPF }\end{array}$ & No si & $\begin{array}{l}\text { gnificant } M \\
\text { e recorded }\end{array}$ & TAs (for DP & I) and ty & $\begin{array}{l}\text { wo significant } \\
\text { respectively }\end{array}$ & YPP & NCPG & R193 & & LG1 & $0.00784^{* *}$ & 15.8 \\
\hline under late sol & wer & ondition. & For MAT $t$ & rait, on & e significant & YPP & NCPE & R232 & & LG3 & $0.048^{*}$ & 19 \\
\hline MTA recorded & on $\mathrm{LC}$ & 54 (under $r$ & hormal cor & dition) & however, no & $\mathrm{HI}$ & CakTr & OSSRO2 & 719 & $.04351^{*}$ & 13.1 & \\
\hline MTA was ident & ifiec & for this tr & t under I & sow & condition. In & $\mathrm{HI}$ & CESSF & R45 & & LG 4 & $0.04655^{*}$ & 5 \\
\hline
\end{tabular}




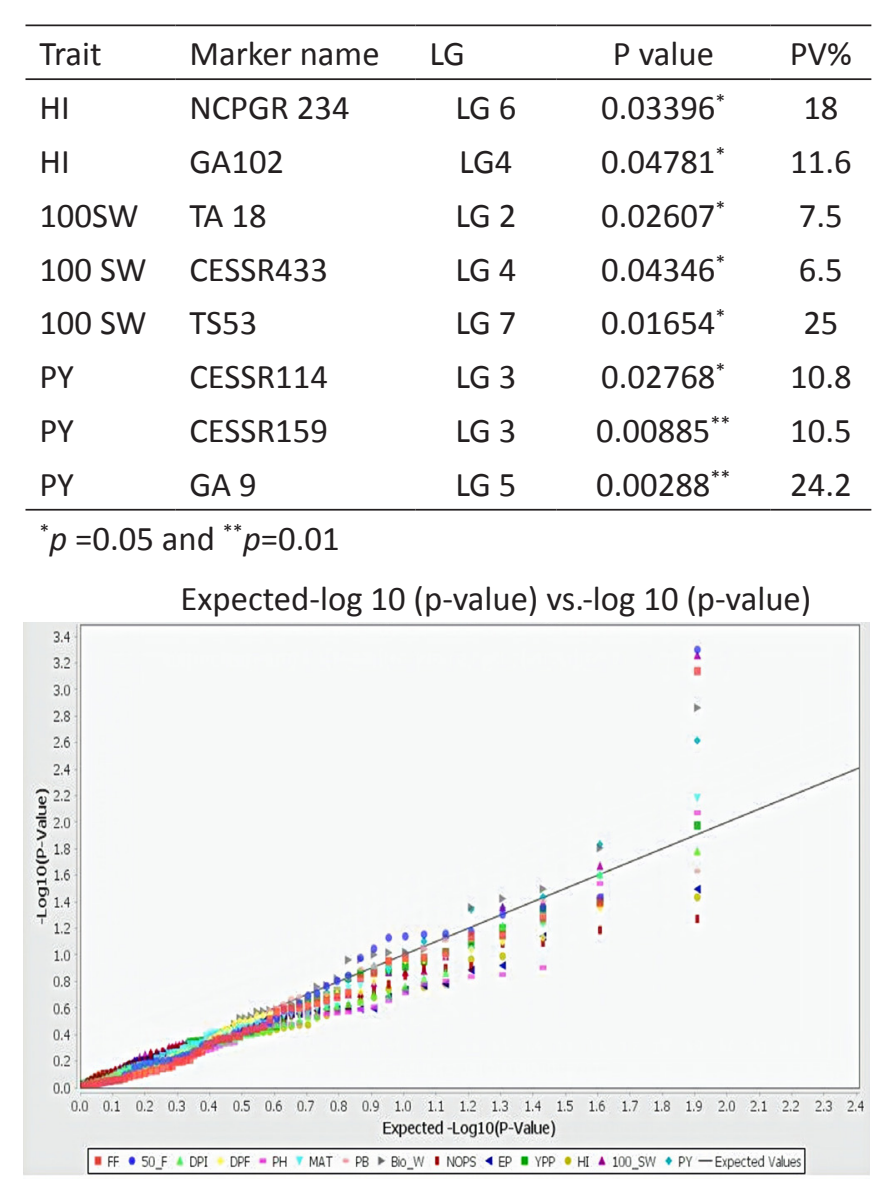

Figure 2: Quantile-Quantile (Q-Q) plots and MTAs distribution for all traits (tested by MLM) under normal sown condition

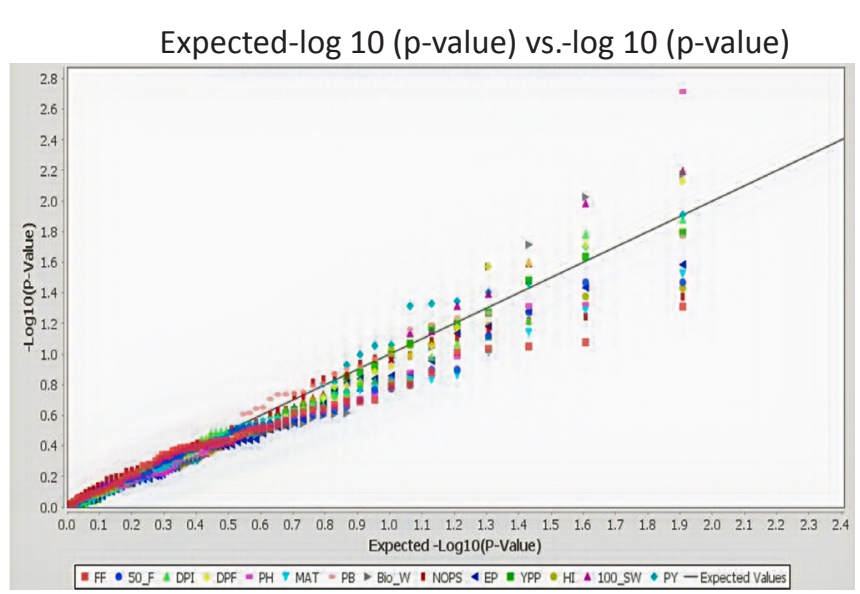

Figure 3: Quantile-Quantile (Q-Q) plots and MTAs distribution for all traits (tested by MLM) under HS sown condition

by Rehman et al. (2011). While considering PH trait three significant MTAs were found on LG1, LG2 and LG4, respectively under HS condition. Likewise two QTLs for same trait were recorded on LG1 and LG4 under drought stress (Rehman et al., 2011). In the context of PH, recently Kale et al. (2015) reported 14 QTL related to PH on LG4 under drought stress. In context of yield related trait,

\begin{tabular}{|c|c|c|c|c|}
\hline Trait & Marker name & LG & $P$ value & PV\% \\
\hline $\mathrm{FF}$ & NCPGR 274 & LG 6 & $0.04892^{*}$ & 15.4 \\
\hline $50 \mathrm{~F}$ & NCPCR 234 & LG 6 & $0.03401^{*}$ & 18.4 \\
\hline $50 \mathrm{~F}$ & TA 64 & LG6 & $0.03402^{*}$ & 20 \\
\hline DPI & NCPGR 234 & LG6 & $0.01656^{*}$ & 21 \\
\hline DPI & NCPGR 274 & LG6 & $0.01321^{*}$ & 22 \\
\hline DPF & GA 9 & LG5 & $0.02674^{*}$ & 19 \\
\hline DPF & NCPGR 234 & LG6 & $0.0257^{*}$ & 19.6 \\
\hline DPF & NCPGR 274 & LG6 & $0.0074^{* *}$ & 21 \\
\hline $\mathrm{PH}$ & TA106 & LG1 & $0.04813^{*}$ & 24.7 \\
\hline PH & NCPGR 202 & LG2 & $0.04712^{*}$ & 19.4 \\
\hline $\mathrm{PH}$ & $\begin{array}{l}\text { C a k Tp S S R } \\
03637\end{array}$ & LG4 & $0.00192^{* *}$ & 30 \\
\hline PB & CESSR 43 & LG 4 & $0.01703^{*}$ & 8 \\
\hline BioY & NCPGR13 & LG1 & $0.01703^{*}$ & 8 \\
\hline BioY & GA105 & LG 5 & $0.00199^{* *}$ & 9 \\
\hline BioY & NCPGR46 & LG7 & $0.048^{*}$ & 8 \\
\hline BioY & STMS & LG6 & $0.048^{*}$ & 7 \\
\hline NOPS & TR 7 & LG 7 & $0.04208^{*}$ & 9 \\
\hline NOPS & TA110 & LG1 & $0.046^{*}$ & 12.7 \\
\hline EP & TA110 & LG 1 & $0.03679^{*}$ & 15 \\
\hline EP & TR 7 & LG 7 & $0.02598^{*}$ & 11 \\
\hline YPP & TA 140 & LG 1 & $0.03472^{*}$ & 10.7 \\
\hline YPP & $\mathrm{H} 5 \mathrm{G} 12$ & LG 6 & $0.01449^{*}$ & 12.8 \\
\hline YPP & TR7 & LG7 & $0.04^{*}$ & 8 \\
\hline YPP & NCPGR202 & LG2 & $0.04^{*}$ & 17.4 \\
\hline YPP & NCPGR156 & - & $0.04^{*}$ & 11.9 \\
\hline $\mathrm{HI}$ & CESSR114 & LG 3 & $0.02806^{*}$ & 10.7 \\
\hline $\mathrm{HI}$ & GA105 & LG 5 & $0.03023^{*}$ & 7 \\
\hline $\mathrm{HI}$ & H1B04 & LG 5 & $0.00975^{* *}$ & 10 \\
\hline $\mathrm{HI}$ & GA26 & LG6 & $0.03923^{*}$ & 15.2 \\
\hline $100 \mathrm{SW}$ & TA18 & LG 2 & $0.01037^{*}$ & 10.1 \\
\hline $100 \mathrm{sW}$ & TA8 & LG 3 & $0.00636^{* *}$ & 22.5 \\
\hline $100 s W$ & NCPGR199 & LG1 & $0.02548^{*}$ & 17.8 \\
\hline $100 \mathrm{sW}$ & TA180 & LG2 & $0.04839^{*}$ & 22.1 \\
\hline $100 s W$ & CakTpSSR03637 & LG4 & $0.04^{*}$ & 17.7 \\
\hline PY & CESSR159 & LG3 & $0.04111^{*}$ & 6 \\
\hline PY & NCPGR 41 & LG 7 & $0.01627^{*}$ & 12.4 \\
\hline PY & TA110 & LG1 & $0.01684^{*}$ & 18.9 \\
\hline PY & TR 7 & LG7 & $0.03999^{*}$ & 9 \\
\hline
\end{tabular}

${ }^{*} p=0.05$ and ${ }^{* *} p=0.01$ 


\begin{tabular}{llccc}
\hline \multicolumn{5}{l}{ Table 6: MTA analysis for various HS indices } \\
\hline Trait & Marker & LG & p value & PV\% \\
& \multicolumn{5}{c}{ groups } \\
\hline HTI & CESSR114 & LG3 & $0.03634^{*}$ & 9.9 \\
HTI & CakTpSSR03637 & LG4 & $0.01813^{*}$ & 17 \\
HTI & GA9 & LG5 & $0.02672^{*}$ & 19.4 \\
YI & TA110 & LG1 & $0.02746^{*}$ & 16.7 \\
YI & CakTpSSR03637 & LG4 & $0.00938^{* *}$ & 19.2 \\
YI & NCPGR41 & LG7 & $0.01833^{*}$ & 12.1 \\
SSI & NCPCR234 & LG6 & $0.04871^{*}$ & 16.9 \\
SSI & STMS25 & LG6 & $0.01865^{*}$ & 12.2 \\
SSI & NCPGR41 & LG7 & $0.00415^{* *}$ & 16.9 \\
MP & NCPGR149 & - & $0.03577^{*}$ & 20.7 \\
MP & CESSR114 & LG3 & $0.0095^{* *}$ & 14.2 \\
MP & CESSR159 & LG3 & $0.00228^{* *}$ & 14.7 \\
MP & GA9 & LG5 & $0.00264^{* *}$ & 19.3 \\
GMP & NCPGR149 & & $0.01926^{*}$ & 17.3 \\
GMP & CESSR114 & LG3 & $0.01571^{*}$ & 12.6 \\
GMP & CESSR159 & LG3 & $0.01028^{*}$ & 10.2 \\
GMP & GA9 & LG5 & $0.01947^{*}$ & 20.8 \\
GMP & TAAS & LG7 & $0.02285^{*}$ & 17.4 \\
\hline "P 0 .05 & & & \\
\hline
\end{tabular}

${ }^{*} p=0.05$ and ${ }^{* *} p=0.01$

Expected-log 10 ( $p$-value) vs.-log 10 ( $p$-value)

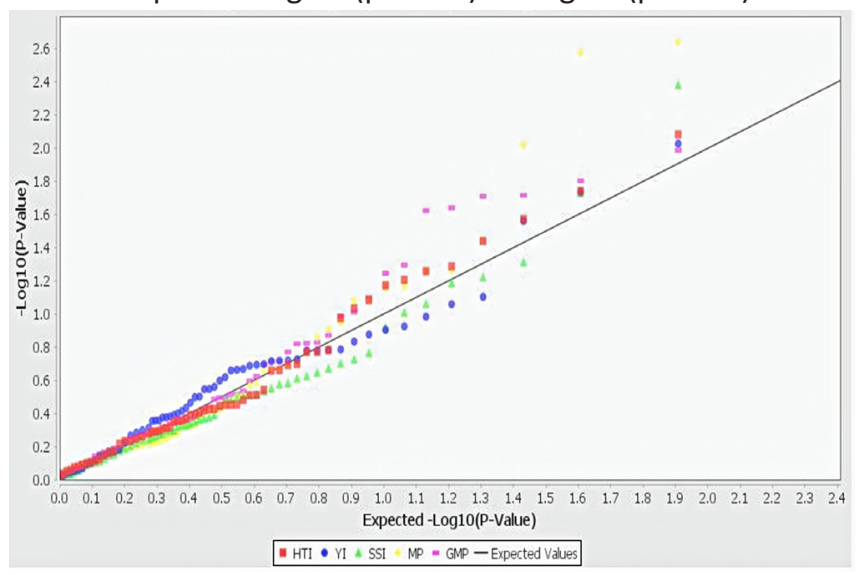

Figure 4:Quantile-Quantile (Q-Q) plots and MTAs distribution for heat tolerance indices (tested by MLM)

four significant MTAs for BioY trait on LG1, LG5, LG6 and LG7, and two significant MTA for NOPS on LG1 and LG7 were noted under late sown condition. Likewise one major QTL governing NOPS on LG7 was registered under salinity stress (Pushpavalli et al., 2015).While Bajaj et al. (2015b) reported several NOPS QTLs existing across all Ca LG (1-8). In another case, four QTLs for NOPS existing on LG1, LG2, LG6 and LG8 were reported (Verma et al., 2015). Based on HI\% trait, we recorded three significant MTAs under both normal and late sown conditions existing on LG3, LG4, LG5 and on LG6. Similarly two QTLs for the $\mathrm{HI}$ trait were found on LG1 and LG3 (Rehman et al., 2011). Importantly, one QTL was registered on LG06 under drought stress for HI trait (Kale et al., 2015). Additionally, a total of six QTLs related to $\mathrm{HI}$ residing across all the LG except LG1 and LG8 has been reported recently (Srivastava et al., 2016).

Considering YPP, five significant MTAs were recorded on LG1, LG3, LG5, LG6 and LG7 under normal condition exhibiting upto $19 \%$ PV, and five significant MTAs on LG1, LG6 and LG7 showing up to $12.8 \% \mathrm{PV}$ were recorded under $\mathrm{HS}$ condition for YPP. Similarly, six YPP related QTLs existing across all the LGs except LG1 and LG8 were reported (Srivastava et al., 2016).

For 100 SW three MTAs harboring on LG2, LG4 and LG7 under normal condition and five significant MTAs under HS located on LG1, LG2, LG3 and LG4 were recorded. Similarly seed wt related QTL located on LG1 (Abbo et al., 2005; Hossain et al., 2010; Gowda et al., 2011), on LG2 (Gowda et al., 2011), on LG4 (Cobos et al., 2007; Abbo et al., 2005; Hossain et al., 2010; Gowda et al., 2011; Jamalabadi et al. 2013; Thudi et al. 2014; Kale et al., 2015) has been reported. Additionally five significant SNPs associated with seed wt were reported on LG1, LG2, LG3 and LG4 (Bajaj et al., 2015a). Subsequently three expression QTLs (e-QTLs) related to seed wt was reported on LG2 and LG7 by Bajaj et al. (2015b). Moreover, Verma et al. (2015) showed seven seed wt QTLs residing on LG1, LG2, LG5, LG6 and LG7. Most importantly, a total of 29 QTLs related to nine different agronomic traits and drought related traits were recovered from QTL-hotspot region on CaLG04 (Kale et al., 2015). Taking note of PY, three significant MTAs under normal and four significant MTAs under late sown conditions lying on LG1, LG3, LG5 and LG7 were obtained in the current study. In this regard three QTLs (related to yield) each was reported to be lying on LG3, LG4 and LG7 under drought stress (Kale et al., 2015).

\subsection{MTA analysis of heat tolerance indices}

Importantly, considering heat tolerant indices viz., $\mathrm{HTI}, \mathrm{YI}, \mathrm{HSI}$, MP, GMP several significant MTAs were recorded (see Table 5). Three significant MTAs were recorded on LG3, LG4 and on LG5.Similarly for YI; three significant MTAs were suggested residing on LG1, LG4 and LG7. Considering SSI a total of 3 MTAs were recorded on LG6 and on LG7. While for MP (4 MTAs) and for GMP (5 MTAs) were noted. Similarly a total of 4 QTLs for drought tolerance index (DTI) harboring on CaLG1, CaLG7 and CaLG8 and one QTL for DST trait on CaLG8 was reported under drought stress (Kale et al., 2015). Thus, these markers significantly associated with various traits could serve as an important repertoire for assisting marker assisted breeding for heat tolerance in chickpea.

\section{Conclusion}

Sufficient amount of genetic variability for various breeding traits was recorded under both normal and HS conditions. 
Thus, the captured genetic variability for various traits under both conditions could be incorporated in breeding programme for improving yield related traits in the high yielding yet HS sensitive chickpea cultivars. Additionally, significant MTAs for various yield related traits could promisingly help facilitating conventional breeding to develop HS tolerant chickpea genotypes via marker assisted selection.

\section{Acknowledgement}

Authors acknowledge support from Indian Council of Agricultural Research (ICAR), India.

\section{Author's Contribution}

Uday Chand Jha conducted the experiment and wrote the manuscript along with Paresh Chandra Kole and Narendra Pratap Singh. The work is part of PhD thesis of the first author. All the authors also thank, Dr. Swarup K Parida, National Institute of Plant genome Research, New Delhi for providing Chickpea SSR markers.

\section{Conflict of Interest}

The authors declare that they have no conflict of interest.

\section{Reference}

Abbo, S., Molina, C., Jungmann, R., Grusak, M.A., Berkovitch, Z., Reifen, R., Kahl, G., Winter, P., Reifen, R., 2005. Quantitative trait loci governing carotenoid concentration and weight in seeds of chickpea (Cicer arietinum L.). Theoretical and Applied Genetics 111, 185-195.

Bajaj, D., Upadhyaya, H.D., Khan, Y., Das, S., Badoni, S., Shree, T., Kumar, V., Tripathi, S., Gowda, C.L.L., Singh, S., Sharma, S., Tyagi, A.K., Chattopdhyay, D., Parida S.K., 2015a. A combinatorial approach of comprehensive QTL-based comparative genome mapping and transcript profiling identified a seed weight-regulating candidate gene in chickpea. Scientific Reports 5, 9264.

Bajaj, D., Saxena, M.S., Kujur, A., Das, S., Badoni, S., Tripathi, S., Upadhyaya, H.D., Gowda, C.L., Sharma, S., Singh, S., Tyagi, A.K., Parida, S.K., 2015b. Genome-wide conserved non-coding microsatellite (CNMS) marker-based integrative genetical genomics for quantitative dissection of seed weight in chickpea. Journal of Experimental Botany 66, 1271-1290.

Bouslama, M., Schapaugh, W.T., 1984. Stress tolerance in soybean, Part I: evaluation of three screening techniques for heat and drought tolerance. Crop Science 24, 933-937.

Bradbury, P.J., Zhang, Z., Kroon, D.E., Casstevens, T.M., Ramdoss, Y., Buckler, E.S., 2007. TASSEL: software for association mapping of complex traits in diverse samples. Bioinformatics 23, 2633-2635.

Choudhary, S., Sethy, N.K., Shokeen, B., Bhatia, S., 2009. Development of chickpea EST-SSR markers and analysis of allelic variation across related species. Theoretical and
Applied Genetics 118, 591-608.

Choudhary, S., Gaur, R., Gupta, S., Bhatia, S., 2012. EST-derived genic molecular markers: development and utilization for generating an advanced transcript map of chickpea. Theoretical and Applied Genetics 124, 1449-1462.

Cobos, M., Rubio, J., Fernandez-Romero, M., Garza, R., Moreno, M.T., Millan, M.T., Gil, J., 2007. Genetic analysis of seed size, yield and days to flowering in a chickpea recombinant inbred line population derived from a Kabuli × Desi cross. Annals of Applied Biology 151, 33-42.

Doyle, J.J., Doyle, J.L., 1987. A rapid DNA isolation procedure for small quantities of fresh leaf tissue, Phytochem Bulletin 19, 11-15.

Devasirvatham, V., Gaur, P., Mallikarjuna, N., Raju, T.N., Trethowan, R.M., Tan, D.K.Y., 2013. Reproductive biology of chickpea response to heat stress in the field is associated with the performance in controlled environments. Field Crops Research 142, 9-19.

Devasirvatham, V., Gaur, P., Mallikarjuna, N., Raju, T.N., Trethowan, R.M., Tan, D.K.Y., 2012. Effect of high temperature on the reproductive development of chickpea genotypes under controlled environments. Functional Plant Biology 39, 1009-1018.

FAOSTAT, 2014. FAO Statistics Division, Available at: http:// faostat3.fao.org/compare/E

(accessed 27.216).

Fischer, R.A., Maurer, R., 1978. Drought Resistance in Spring Wheat Cultivars, I. Grain Yield Response. Australian Journal of Agriculture Research 29, 897-912.

Federer, W.T., 1956. Augumented (or Hoonuiaku) design. Hwaaiian Planters Record, 55, 191-208.

Fernandez, G.C.J., 1992. Effective selection criteria for assessing plant stress tolerance. In: Kus, E.G. (Ed.), Adaptation of Food Crop Temperature and Water Stress, Proceeding of $4^{\text {th }}$ International Symposium, Asian Vegetable and Research and Development Center, Shantana, Taiwan, 257-270.

Gaur, R., Sethy, N.K., Choudhary, S., Shokeen, B., Gupta, V., Bhatia, S., 2011. Advancing the STMS genomic resources for defining new locations on the intraspecific genetic linkage map of chickpea (Cicer arietinum L.). BMC Genomics 12, 117.

Gowda, S., Radhika, P., Mhase, L., Jamadagni, B., Gupta, V., Kadoo, N., 2011. Mapping of QTLs governing agronomic and yield traits in chickpea. Journal of Applied Genetics 52, 9-21.

Graham, P.H., Vance, C.P., 2003. Legumes: Importance and Constraints to Greater Use. Plant Physiology 131,872877.

Hossain, A.B.S., Sears, A.G., Cox, T.S., Paulsen, G.M., 1990. Dessication tolerance and its relationship to assimilate partitioning in winter wheat. Crop Science 30, 622-627.

Hossain, S., Ford, R., McNeil, D., Pittock, C., Panozzo, J., 2010. Inheritance of seed size in Cicer arietinum L. and identification of QTL based on 100 seed weight and seed 
size index. Australian Journal of Crop Science 4, 126-135. Jamalabadi, J.G., Saidi, A., Karami, E., Kharkesh, M., Talebi, R., 2013. Molecular Mapping and Characterization of Genes Governing Time to Flowering, Seed Weight, and Plant Height in an Intraspecific Genetic Linkage Map of Chickpea (Cicer arietinum). Biochemical Genetics 51, 387-397.

Jha, U.C., Bohra, A., Singh, N.P., 2014a. Heat stress in crop plants: its nature, impacts and integrated breeding strategies to improve heat tolerance. Plant Breeding $133,679-70$.

Jha, U.C., Chaturvedi, S.K., Bohra, A., Basu, P.S., Khan, M.S., Barh, D., 2014b. Abiotic stresses, constraints and improvement strategies in chickpea. Plant Breeding 133, 163-178.

Jha, U.C., Shil, S., 2015. Association Analysis of yield contributing traits of chickpea genotypes under high temperature condition. Trends in Bioscience 8, 23352341.

Jha, U.C., Basu, P.S., Singh, D.K., 2015 Genetic variation and diversity analysis of chickpea genotypes based on quantitative traits under high temperature stress. International Journal of Bio-resource Stress Management 6, 700-706.

Jha, U.C., Bohra, A., Jha, R., Parida, S., 2017. Integrated 'omics' approaches to sustain major global grain legume productivity under heat stress. Plant Breeding 136, 437-59.

Jha, U.C., Jha, R., Bohra, A., Parida, S.K., Kole, P.C., Thakro, V., Singh, D., Singh, N.P., 2018. Population structure and association analysis of heat stress relevant traits in chickpea (Cicer arietinum L.). 3 Biotech 8, 43.

Kale, S.M., Jaganathan, D., Ruperao, P., Chen, C., Punna, R., Kudapa, H., Thudi, M., Roorkiwal, M., Katta, M.A., Doddamani, D., Garg, V., Kishor P.B., Gaur, P.M., Nguyen, H.T., Batley, J., Edwards, D., Sutton, T., Varshney, R.K., 2015. Prioritization of candidate genes in "QTL-hotspot" region for drought tolerance in chickpea (Cicer arietinum L.). Scientific Reports 5, 15296.

Kalra, N., Chakraborty, D., Sharma, A., Rai, H.K., Jolly, M., Chander, S., Kumar, P.R., Bhadraray, S., Barman, D., Mittal, R.B., Lal, M., Sehgal, M., 2008. Effect of temperature on yield on some winter crops in northwest India. Current Science 94, 82-88.

Krishnamurthy, L., Gaur, P.M., Basu, P.S., Chaturvedi, S.K., Tripathi, S., Vadez, V., Rathore, A., Varshney, R.K., Gowda, C.L.L., 2011. Large genetic variation for heat tolerance in the reference collection of chickpea (Cicer arietinum L.) germplasm. Plant Genetic Resources 9, 59-69.

Pushpavalli, R., Krishnamurthy, L., Thudi, M., Gaur, P.M., Rao, M.V., Siddique, K.H.M, Colmer, T.D., Turner, N.C., Varshney, R.K., Vadez, V., 2015. Two key genomic regions harbour QTLs for salinity tolerance in ICCV $2 \times$ JG 11 derived chickpea (Cicer arietinum L.) recombinant inbred lines. BMC Plant Biology 15, 124.

Rehman, A.U., Malhotra, R.S., Bett, K., Tar'an, B., Bueckert, R., Warkentin, T.D., 2011. Mapping QTL Associated with Traits Affecting Grain Yield in Chickpea (L.) under Terminal Drought Stress. Crop Science 51, 450-463.

Sethy, N.K., Shokeen, B., Bhatia, S., 2003. Isolation and characterization of sequence-tagged microsatellite sites markers in chickpea (Cicer arietinum L.). Molecular Ecology Notes 3, 428-430.

Sethy, N. K., Shokeen,. B, Edwards, K.J., Bhatia, S., 2006. Development of microsatellite markers and analysis of intra specific genetic variability in chickpea (Cicer arietinum L.). Theoretical and Applied Genetics 112, 1416-1428.

Srivastava, R., Bajaj, D., Malik, A., Singh, M., Parida, S.K., levantmolecular tags regulating agronomic traits in chickpea. Scientific Reports 6, 33616.

Thudi, M., Upadhyaya, H.D., Rathore, A., Gaur, P.M., Krishnamurthy, L., Roorkiwal, M., Spurthi N. Nayak, S.N., Chaturvedi, S.K., Basu, P.S., Gangarao, N.V.P.R., Firke, A., Kimurto, P., Sharma, P.C., Sheshashayee, M.S., Tobota, S., Kashiwagi, J., Ito, O., Killian, A., Varshney, R.K., 2014. Genetic Dissection of Drought and Heat Tolerance in Chickpea through Genome-Wide and Candidate GeneBased Association Mapping Approaches. PLoS ONE 9, e96758.

Varshney, R.K., Thudi, M., Nayak, S.N., Gaur, P.M., Kashiwagi, J., Krishnamurthy, L., Jaganathan, D., Koppolu, J., Bohra, A., Tripathi, S., Rathore, A., Jukanti, A.K., Jayalakshmi, V., Vemula, A., Singh, S.J., Yasin, M., Sheshshayee, M.S., Viswanatha, K.P., 201 4. Genetic dissection of drought tolerance in chickpea (Cicer arietinum L.). Theoretical and Applied Genetics 127, 445-462.

Verma, S., Gupta, S., Bandhiwal, N., Kumar, T., Bharadwaj, C., Bhatia, S., 2015. High-density linkage map construction and mapping of seed trait QTLs in chickpea (Cicer arietinum L.) using Genotyping-by- Sequencing (GBS). Scientific Reports 5, 17512. 